\title{
A Novel Micro Optical Probe for Early Diagnosis of Upper Gastrointestinal (GI) Cancers
}

\author{
A. Garcia-Uribe ${ }^{1 *}$, K. C. Balareddy ${ }^{1}$, J. Zou ${ }^{1}$, K. K. Wang ${ }^{2}$, and L. V. Wang ${ }^{3}$ \\ ${ }^{1}$ Department of Electrical and Computer Engineering, Texas A\&M University, College Station, Texas, USA \\ ${ }^{2}$ Mayo Clinic, Division of Gastroenterology and Hepatology, Rochester, Minnesota, USA \\ ${ }^{3}$ Department of Biomedical Engineering, Washington University in St. Louis, St. Louis, Missouri, USA \\ "correspondence: aguribe@tamu.ediu
}

\begin{abstract}
In this paper, we present the development of a new micro fiber optical sensor probe with side viewing capability for early diagnosis of cancers in upper gastrointestinal tract. The probe consists of a oblique incidence source and a collection array of microfabricated curved SU-8 waveguides for collecting spatially resolved diffuse reflectance from tissue surface. The probe has been used to measure ex-vivo esophagus specimens to differentiate cancerous tissues from benign ones.
\end{abstract}

\section{INTRODUCTION}

The American Cancer Society estimates that cancer of the esophagus affects about 16,470 people with about 14,280 deaths each year in the United States [1]. There are two main types of esophageal cancer: squamous cell carcinoma and adenocarcinoma. The esophagus is normally lined with squamous cells and most squamous cell cancers occur in the mid to proximal esophagus. Adenocarcinomas start in columnar tissue, which can be found in the distal esophagus. If Barrett's esophagus occurs in an intestinal area that previously contained squamous cells, it could develop into adenocarcinoma.

Several different noninvasive methods for optical and spectroscopic methods for tissue diagnosis have been proposed to detect the malignancy of tumors [2]-[4],[5]. Oblique incidence diffuse reflectance spectroscopy (OIDRS) is a novel non-invasive optical method for early diagnosis of epithelial types of cancer, which utilizes an optical fiber sensor probe to conduct upon contact comparative measurements of the spatially resolved diffuse reflectance spectra of interested tissues [6]. To significantly extend OIDRS for the detection of cancers inside human body, new sensor probes with much smaller size and more importantly side viewing capability are necessary.

\section{OBLIQUe InCIDENCE DIFFUSE REFLECTANCE SPECTROSCOPY}

When light is incident on the surface of an inhomogeneous media (e.g. biological tissue), part of the incident light will be directly reflected (specular reflectance) and the remaining will transmit into and interacts with the media. The diffuse reflectance is the portion of the light that after undergoing multiples interactions with the media returns and escapes from the surface (Fig. 1).

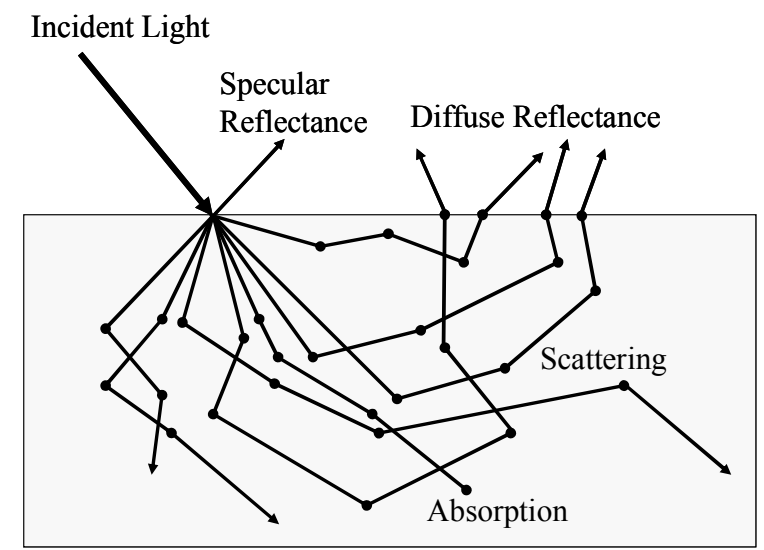

Fig, 1. Illustration of the light interaction in a scattering and absorption media

The spatially resolved steady-state diffuse reflectance for particular wavelength and oblique incidence can be calculated by [7].

$$
\begin{array}{r}
R(r)=\left(1-R_{s}\right) a \cdot\left[\frac{z^{\prime}\left(1+\mu_{e f f} \rho_{1}\right) \exp \left(-\mu_{e f f} \rho_{1}\right)}{4 \pi \rho_{1}^{3}}+\right. \\
\left.\frac{\left(z^{\prime}+2 z_{b}\right)\left(1+\mu_{e f f} \rho_{2}\right) \exp \left(-\mu_{e f f} \rho_{2}\right)}{4 \pi \rho_{2}^{3}}\right]
\end{array}
$$

where $R_{\mathrm{s}}$ is the specular reflection, $a^{\prime}$ is albedo, $r$ is the distance between the point of observation on the tissue surface and the point of incidence, $z$ ' is the distance between the virtual boundary and the tissue depth, $z_{\mathrm{b}}$ is the distance between the virtual boundary and the tissue surface, and $\rho_{1}$ and $\rho_{2}$ are the distances between the point sources and the point of observation (Fig 2). The effective attenuation 
coefficient $\mu_{e f f}=\left(\mu_{a} / D\right)^{1 / 2}$. For oblique incidence the diffusion coefficient [8]

$$
D=\frac{1}{3\left(0.35 \mu_{a}+\mu_{s}^{\prime}\right)}
$$

The shift $\Delta \mathrm{x}$ of the point sources in the $x$ direction is

$$
\Delta x=\frac{\sin \left(\alpha_{t}\right)}{0.35 \mu_{a}+\mu_{s}{ }^{\prime}}
$$

where $\alpha_{t}$ is the angle of light transmission into the tissue. The absorption and reduced scattering coefficients can be calculated by.

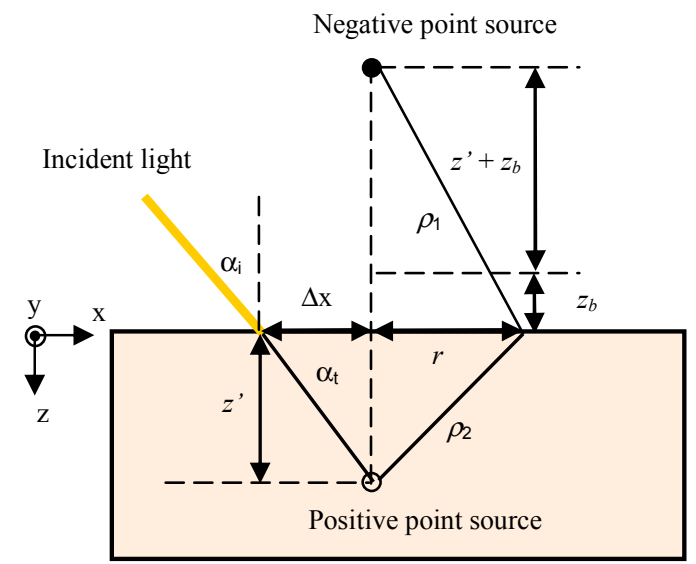

Fig. 2. Schematic of the diffusion theory model for oblique incidence (incidence plane).

Applying nonlinear least squares fit to the reflectance expression yields the effective attenuation coefficient $\left(\mu_{\mathrm{eff}}\right)$. The absorption coefficient $\mu_{a}$ and is the reduced scattering coefficient $\mu_{s}^{\prime}$ are calculated by

$$
\begin{gathered}
\mu_{a}=\frac{\mu_{e f f}^{2} \Delta x}{3 \sin \left(\alpha_{t}\right)} \\
\mu_{s}{ }^{\prime}=\frac{\sin \left(\alpha_{t}\right)}{\Delta x}-0.35 \mu_{a}
\end{gathered}
$$

The diffusion equation assumes that the reduced scattering coefficient is much larger than the absorption. The source and detector must also be separated in space so that the light is diffuse when it reaches the detector. When the distance between the source and the detectors is comparable to the transport mean free path, diffusion theory does not apply. In this case, Monte Carlo simulation can be applied for the extraction of optical properties from the measured diffuse reflectance [9].

\section{PRobe Design And FABrication}

New sensor probes with much smaller size and more importantly side viewing capability are necessary for effective OIDRS measurement in the narrow of the esophagus (Fig. 3). However, achieving probe miniaturization and side-viewing capability requires dense placement and sharp bending of optical fibers, which would cause excessive light loss, cross-talk or breaking of the fiber. To overcome these problems, an entire new device design and structure were developed.

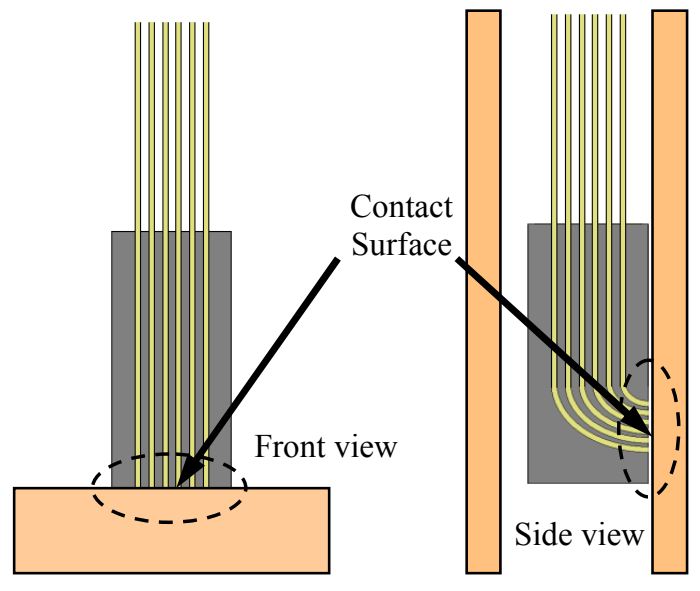

(a)

Fig, 3. Schematic of OIDRS probe configuration: (a) front viewing and (b) side viewing

To conduct OIDRS measurement, it is necessary to accurately deliver light of particular wavelength at a desirable oblique incidence angle on the tissue surface and also collect the one-dimensional linear distribution of the diffuse reflectance $\mathrm{R}(\mathrm{x})$. While this can be achieved by placing the biological sample under an imaging setup, it will not be convenient and feasible to conduct in-vivo OIDRS measurements under ordinary clinical settings. Apart from the oblique incidence fibers, all other fibers remain straight without any need of bending, such that they can be arranged in a dense manner without possibility of fracture. However, the measurements from tissue inside human body, (such as esophagus) require a different configuration that allows the further miniaturization of the sensor and more importantly the side-view capability or the sensor, in which all the collection fibers have to undergo a sharp $90^{\circ}$ turn within a tight space within $2 \sim 5 \mathrm{~mm}$. This inevitably would cause significant light loss and leakage and also possible mechanical fracture of the collection fibers. To address this problem, we have designed a probe which consists of three micromachined silicon substrates (Fig. 4). The first substrate consists of a guiding structure made of $100 \mu \mathrm{m}$ thick SU-8 layer, which serves as a position device for the source fiber to provide light incidence at an oblique angle $\left(45^{\circ}\right)$ (Fig. 5). The source fiber with a diameter of $200 \mu \mathrm{m}$ can be accommodated using a matching pair of two positioning substrates. The second substrate carries ten SU-8 waveguides $\left(100 \times 100 \mu \mathrm{m}^{2}\right)$ with a $90^{\circ}$ turn for collecting the spatially 
resolved diffuse reflectance from the tested tissue surface (Fig. 5). The third substrate consists of a 1-dimensional array of bulk-etched $\mathrm{V}$-groves to ensure precise position of the 10 interconnection fibers to interface the $90^{\circ}$ collection waveguides to OIDRS measurement setup (Fig. 5). We choose SU-8 as structural materials for both the guiding structures of source fiber and also the curved waveguides for collecting the diffuse reflectance. SU-8 is preferable since it can be directly photolithographically patterned to form thick microstructures $(100 \mu \mathrm{m})$ without further etching process, which results in a straightforward and low-cost fabrication process. SU-8 has high optical transparency above $400 \mathrm{~nm}$. The Fig. 6 shows the Optical absorbance of SU-8. The top/bottom surfaces and the two side walls of the SU-8 waveguides were coated with evaporated aluminum $(180 \mathrm{~nm}$ thick). To ensure a good coverage of the two side walls and the top surface, the aluminum depositions were conducted with the substrate placed at an oblique $\pm 20^{\circ}$ with respect to the evaporation source.
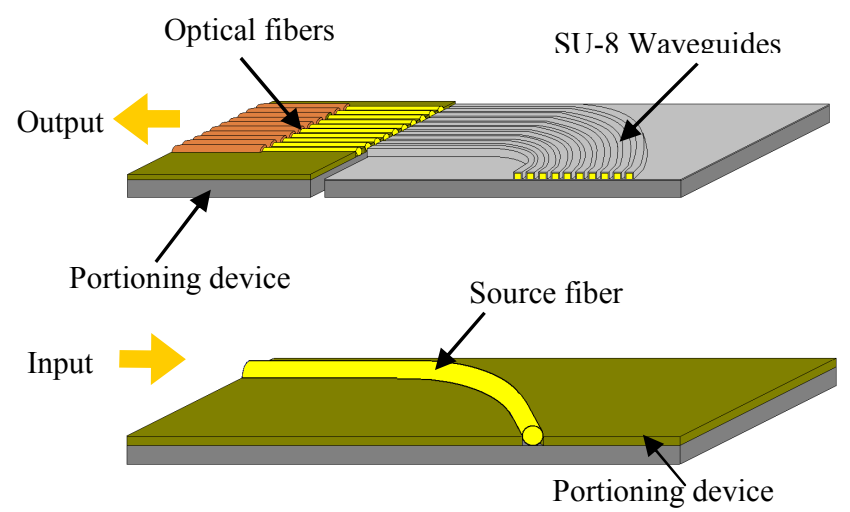

Fig. 4. Schematic design of the side-viewing OIDRS probe.

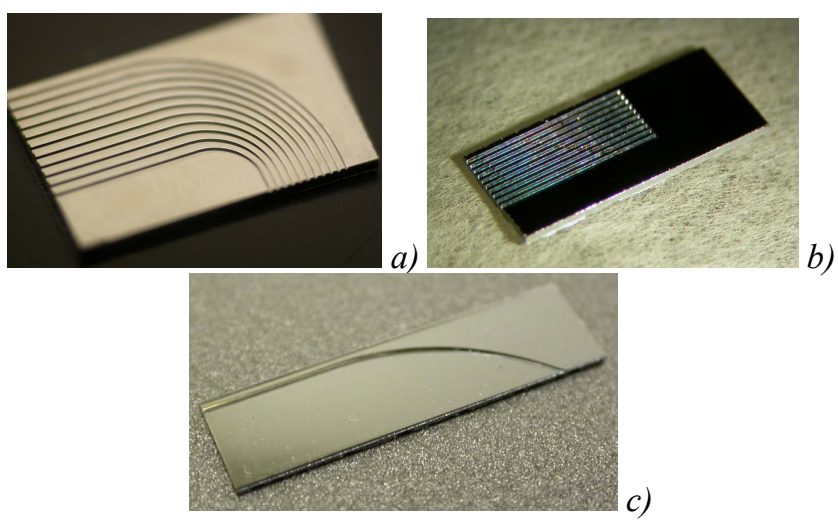

Fig. 5. Micromachined silicon positioning substrates for the OIDRS probe (a) Source fiber guide; b) Collection waveguide substrates; and c) Interconnection fiber guide.

After the fabrication of three substrates, both the source fiber and the interconnection fibers were carefully placed into their positioning substrates and fixed in place with black epoxy. Next, all the substrates were aligned and glue together to complete the assembly of the entire probe in an aluminum holder for protection (Fig. 7).

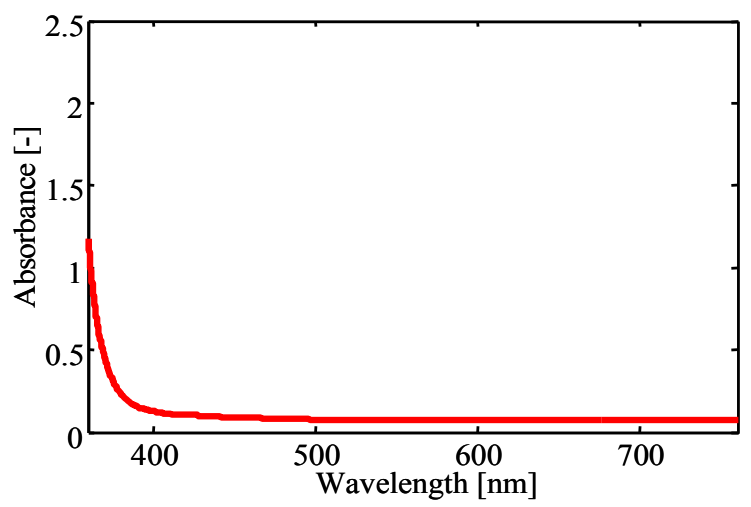

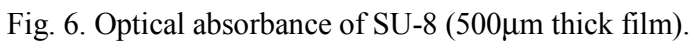

The outer dimension of the sensor head is $5 \times 5 \times 12 \mathrm{~mm}^{3}$, which is small enough for esophagus inspection. The source and collection fibers of the sensor probe were then connected to the system via SMA connectors (Fig. 7).

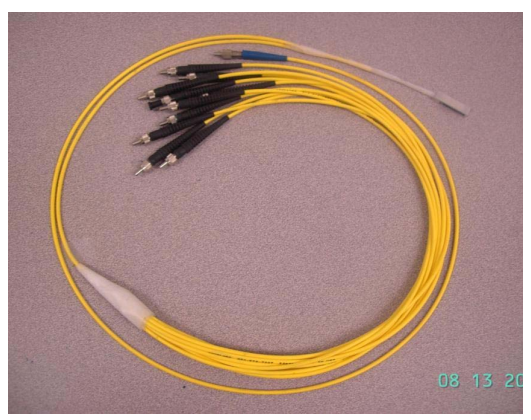

Fig. 7. Complete OIDRS probe.

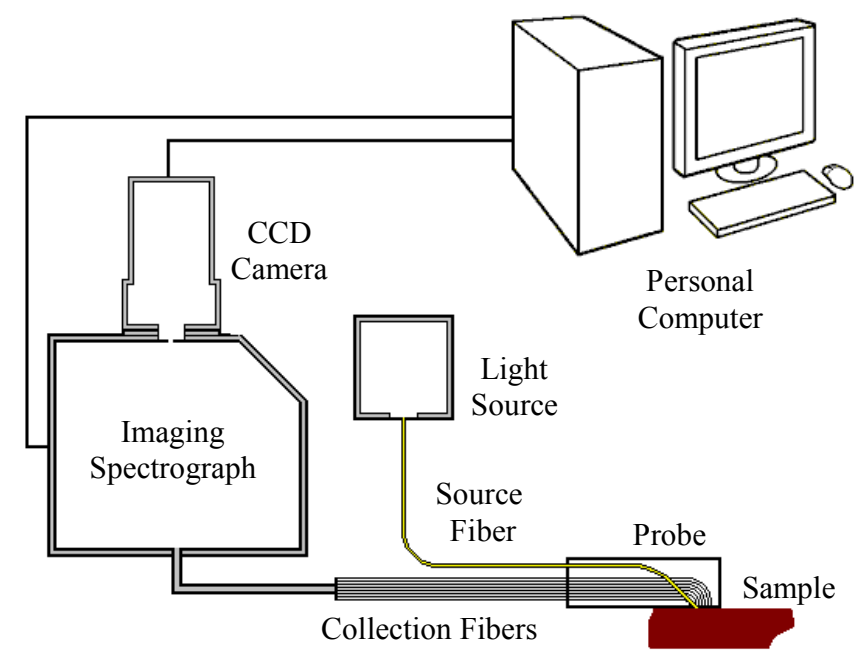

Fig. 8 Experimental setup for OIDRS probe testing. 


\section{OIDRS SYSTEM}

The Fig. 8 shows the Experimental OIDRS setup. White light from a halogen lamp is coupled to a single optical fiber (source fiber) that delivers light to the skin area of interest. The sensor probe collects the diffuse reflected light from the surface of the sample. The optical fibers aligned by the connecting interface are coupled to a bundle placed in the object plane of the imaging spectrograph. The spectrograph generates an optical spectrum for each optical fiber and projects the spectra onto a CCD camera from the wavelength range of 455 to $765 \mathrm{~nm}$. The steady-state diffuse reflectance spectra from each collection fiber are stored in the computer for further analysis.

\section{EXPERIMENTAL RESULTS}

Before the measurements were performed, the OIDRS system was calibrated using a standard liquid phantom, which consists of trypan blue dyes as the absorbers and polystyrene microspheres as the scattering elements [10].

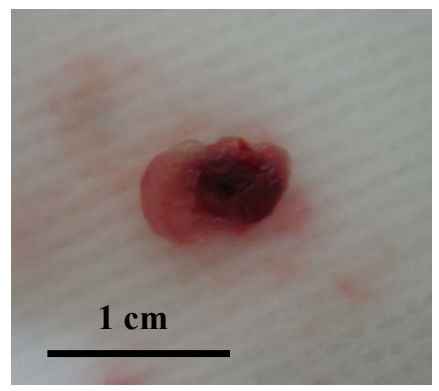

Fig.. 9. An esophageal biopsy sample

After the calibration, the entire OIDRS system was used to conduct ex-vivo recently biopsied esophagus specimens (Fig. 9). The measurement results by the ten collection channels clearly show different diffuse reflectance spectra for the normal and cancerous tissues (Fig. 10). The general lower diffuse reflectance of the cancerous tissue is caused by its darker color (causing more light absorption). The stronger scattering of the cancerous tissue is believed to be caused by the relatively larger cell nuclei as stronger optical scatter in malignant tumor cells, which is expected to serve as a good indicator to effectively differentiate cancerous tissue from benign ones [11].

\section{CONCLUSIONS}

A new miniaturized optical sensor probe for oblique incidence diffuse reflectance spectroscopy has been developed and used for ex-vivo testing to differentiate benign samples from esophageal caner. These results are highly promising and indicate that the approach presented can potentially be used with medical endoscopes to enable non-invasive in-vivo detection of esophageal cancer and other epithelial types of cancers.
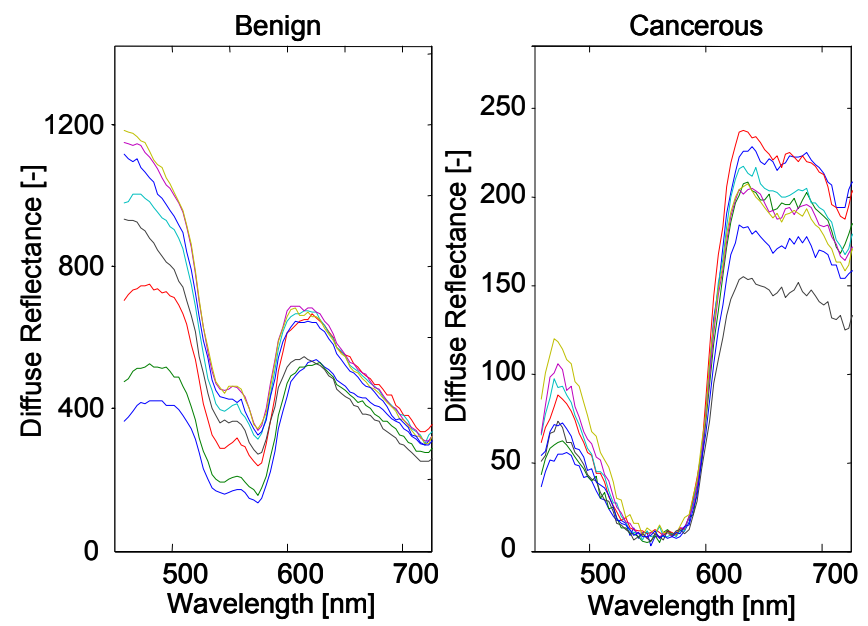

Fig. 10. Ex-vivo OIDRS measurement: (a) Normal tissue; and (b) Cancer tissue.

\section{ACKNOWLEDGMENTS}

This project is supported by National Institutes of Health R01 CA106728.

\section{REFERENCES}

[1] American Cancer Society, Cancer Facts \& Figures 2008 [Online], http://www.cancer.org.

[2] I. Georgakoudi et al., "Fluorescence, reflectance, and "lightscattering spectroscopy for evaluating dysplasia in patients with Barrett's esophagus," Gastroenterology, vol. 120, pp. 1620-1629, 2001

[3] P. R. Bargo et al., "In vivo determination of optical properties of normal and tumor tissue with white light reflectance and an empirical light transport model during endoscopy," J. Biomed. Opt., vol. 10, $034018,2005$.

[4] S. Brand et al., "Optical Coherence Tomography in the Gastrointestinal Tract," Endoscopy 32, pp. 796-803, 2000.

[5] H. Messmann, et al., "Endoscopic fluorescence detection of dysplasia in patients with Barrett's esophagus, ulcerative colitis, or adenomatous polyps after 5-aminolevulinic acid-induced protoporphyrin IX sensitization," Gastrointestinal Endoscopy, 49(1), pp. 97-101, 1999.

[6] A. Garcia-Uribe et al., "Skin cancer detection by spectroscopic oblique-incidence reflectometry: classification and physiological origins," Appl. Opt., vol. 43, pp. 2643-2650, 2004.

[7] L.-H. Wang and S. L. Jacques, "Use of a laser beam with an oblique angle of incidence to measure the reduced scattering coefficient of a turbid medium," Appl. Opt., vol. 34, pp. 2362-2366, 1995

[8] S.-P. Lin, L.-H. Wang, S. L. Jacques, and F. K. Tittel, "Measurement of tissue optical properties using oblique incidence optical fiber reflectometry," Appl. Opt. vol. 36, pp. 136-143, 1997.

[9] A. Kienle and M. S. Patterson, "Determination of the optical properties of turbid media from a single Monte Carlo simulation," Phys. Med. Biol. vol. 41, pp. 2221-2227, 1996.

[10] G. Marquez and L.-H. Wang, "White light oblique incidence reflectometer for measuring absorption and reduced scattering spectra of tissue-like turbid media," Opt. Express, vol. 1, 454-460 1997.

[11] L. T. Perelman et al., "Observation of periodic fine structure in reflectance from biological tissue-a new technique for measuring nuclear size distribution," Phys. Rev. Lett., vol. 80, pp. 627-630, 1998. 